TRANSACTIONS OF THE

AMERICAN MATHEMATICAL SOCIETY

Volume 362, Number 10, October 2010, Pages 5043-5062

S 0002-9947(10)04894-4

Article electronically published on May 17, 2010

\title{
RESULTS ON A WEIGHTED POINCARÉ INEQUALITY OF COMPLETE MANIFOLDS
}

\author{
KWAN-HANG LAM
}

\begin{abstract}
We study manifolds satisfying a weighted Poincaré inequality, which was first introduced by $\mathrm{Li}$ and Wang. We generalized their result by relaxing the Ricci curvature bound condition only being satisfied outside a compact set and established a finitely many ends result. We also proved a vanishing result for an $L^{2}$ harmonic 1-form provided that the weight function $\rho$ is of sub-quadratic growth of the distance function, which generalized the Li-Wang result on manifolds with a positive spectrum.
\end{abstract}

\section{INTRODUCTION}

In a work of Witten and Yau [13, the authors proved that if $M^{n}$ is a conformally compact, Einstein, $n$-dimensional manifold $(n \geq 3)$ whose boundary has positive Yamabe constant, then $M$ must have only one end. Shortly after that, Cai and Galloway [1] relaxed the assumptions to allow the boundary of $M$ to have nonnegative Yamabe constant. In [12, X. Wang proved the following:

Theorem (X. Wang). Let $M^{n}$ be an $n$-dimensional $(n \geq 3)$, conformally compact manifold with Ricci curvature bounded from below by

$$
\operatorname{Ric}_{M} \geq-(n-1) .
$$

Let $\lambda_{1}(M)$ be the lower bound of the spectrum of the Laplacian on $M$. If

$$
\lambda_{1}(M) \geq n-2
$$

then either

(a) $H^{1}\left(L^{2}(M)\right)=0$; or

(b) $M=\mathbb{R} \times N$ with the warped product metric $d s^{2}=d t^{2}+\cosh ^{2} t d s_{N}^{2}$, where $N$ is a compact manifold with Ric $_{N} \geq n-2$. In particular, $M$ either has only one end or it must be a warped product given as above.

The above result generalized the results of Witten and Yau and Cai and Galloway since a theorem of Mazzeo [10] identifies the $L^{2}$ cohomology group $H^{1}\left(L^{2}(M)\right)$ with the relative cohomology group $H^{1}(M, \partial M)$ for conformally compact manifolds and

Received by the editors December 17, 2007.

2000 Mathematics Subject Classification. Primary 54C40, 14E20; Secondary 46E25, 20C20.

Key words and phrases. Weighted Poincaré inequality, parallel forms.

This research was partially supported by NSF grant \#0503735 and NSC grant 96-2115-M-006017 of the ROC. 
a theorem of Lee [4] asserts that $\lambda_{1}(M)=\frac{(n-1)^{2}}{4}$ for an $n$-dimensional Einstein, conformally compact manifold with nonnegative Yamabe constant for its boundary. In [8], Li and Wang considered a complete $n$-dimensional Riemannian manifold $M^{n}$ with Ricci curvature bounded from below by

$$
\operatorname{Ric}_{M} \geq-\frac{n-1}{n-2} \lambda_{1}(M),
$$

where $\lambda_{1}(M)$, the greatest lower bound of the spectrum of the Laplacian acting on $L^{2}$ functions, is assumed to be positive. They proved the following.

Theorem (Li-Wang). Let $M^{n}$ be a complete Riemannian manifold of dimension $n \geq 3$. Suppose $\lambda_{1}(M)>0$ and

$$
\operatorname{Ric}_{M} \geq-\frac{n-1}{n-2} \lambda_{1}(M)
$$

Then either

(1) $M$ has only one end with infinite volume; or

(2) $M=\mathbb{R} \times N$ with the warped product metric

$$
d s_{M}^{2}=d t^{2}+\cosh ^{2}\left(\sqrt{\frac{\lambda_{1}(M)}{n-2}} t\right) d s_{N}^{2},
$$

where $N$ is a compact manifold with Ricci curvature bounded from below by

$$
\operatorname{Ric}_{N} \geq-\lambda_{1}(M) \text {. }
$$

Since all the ends of a conformally compact manifold must have infinite volume, the above theorem thus generalized the work of [1, [12] and [13] to complete manifolds with positive spectrum. Since $\lambda_{1}(M)>0$, the variational principle for $\lambda_{1}(M)$ implies the following Poincaré inequality:

$$
\lambda_{1}(M) \int_{M} \phi^{2} \leq \int_{M}|\nabla \phi|^{2},
$$

for any compactly supported smooth function $\phi \in C_{c}^{\infty}(M)$. In [9], the authors considered manifolds satisfying a weighted Poincaré inequality and generalized most of their results in [8] to manifolds satisfying a weighted Poincaré inequality. A manifold $M^{n}$ is said to satisfy a weighted Poincaré inequality with a nonnegative weight function $\rho(x)$ if

$$
\int_{M} \rho(x) \phi^{2}(x) d V \leq \int_{M}|\nabla \phi|^{2} d V,
$$

for any compactly supported smooth function $\phi \in C_{c}^{\infty}(M)$. In particular, when $\rho(x)=\lambda_{1}(M)$ is a positive constant, $M$ is a manifold with positive spectrum. We say that a manifold $M$ has property $\left(\mathcal{P}_{\rho}\right)$ if a weighted Poincaré inequality is valid on $M$ with some nonnegative weight function $\rho$ and the $\rho$-metric, defined by

$$
d s_{\rho}^{2}=\rho d s_{M}^{2}
$$

is complete. Define

$$
S(R)=\sup _{B_{\rho}(R)} \sqrt{\rho},
$$


where $B_{\rho}(R)$ is the ball of radius $R$ (with respect to some fixed point $p$ ) under the $\rho$-metric. In [9], the authors proved the following.

Theorem 0.1 (Li-Wang). Let $M^{n}$ be a complete manifold with dimension $n \geq 3$. Assume that $M$ satisfies property $\left(\mathcal{P}_{\rho}\right)$ for some nonzero weight function $\rho \geq 0$. Suppose

$$
\operatorname{Ric}_{M}(x) \geq-\frac{n-1}{n-2} \rho(x)
$$

for all $x \in M$. If $\rho$ satisfies the growth estimate

$$
\liminf _{R \rightarrow \infty} \frac{S(R)}{F(R)}=0,
$$

where

$$
F(R)= \begin{cases}\exp \left(\frac{n-3}{n-2} R\right) & \text { for } n \geq 4, \\ R & \text { for } n=3\end{cases}
$$

then either

(1) $M$ has only one nonparabolic end; or

(2) $M$ has two nonparabolic ends and is given by $M=\mathbb{R} \times N$ with the warped product metric

$$
d s_{M}^{2}=d t^{2}+\eta^{2}(t) d s_{N}^{2},
$$

for some positive function $\eta(t)$, and some compact manifold $N$. Moreover, $\rho(t)$ is a function of $t$ alone satisfying

$$
\eta^{\prime \prime} \eta^{-1}=\rho
$$

and

$$
\liminf _{x \rightarrow \infty} \rho(x)>0 ; \text { or }
$$

(3) $M$ has one parabolic end and one nonparabolic end and is given by $M=$ $\mathbb{R} \times N$ with the warped product metric

$$
d s_{M}^{2}=d t^{2}+\eta^{2}(t) d s_{N}^{2},
$$

for some positive function $\eta(t)$, and some compact manifold $N$. Moreover, $\rho(t)$ is a function of $t$ alone satisfying

$$
\eta^{\prime \prime} \eta^{-1}=\rho
$$

and

$$
\liminf _{x \rightarrow \infty} \rho(x)>0 \text { on the nonparabolic end. }
$$

It is interesting to see if a similar theorem holds by relaxing the above assumptions to be only satisfied outside a compact set of $M$. In this article, the following theorem has been established:

Theorem 0.2. Let $M^{n}$ be a complete manifold with dimension $n \geq 3$. Assume that $M$ has property $\left(\mathcal{P}_{\rho}\right)$. Also assume that the Ricci curvature of $M$ satisfies the lower bound

$$
\operatorname{Ric}_{M \backslash K}(x) \geq-\frac{n-1}{n-2} \rho(x)+\tilde{\varepsilon}
$$


for some $\tilde{\varepsilon}>0$, compact set $K \subseteq M$. If $\rho$ satisfies the growth estimate

$$
\liminf _{R \rightarrow \infty} \frac{S(R)}{F(R)}=0
$$

where

$$
F(R)= \begin{cases}\exp \left(\frac{n-3}{n-2} R\right) & \text { for } n \geq 4, \\ R & \text { for } n=3,\end{cases}
$$

then $M$ has only finitely many nonparabolic ends. If in addition $\liminf _{x \rightarrow \infty} \rho(x)>0$, then $M$ has only finitely many infinite volume ends.

Remark 0.3. One may assume $\rho$ is positive somewhere outside $K$; otherwise $M$ will have positive Ricci curvature outside a compact set and the conclusion is well known (see [9], for instance).

On the other hand, Li and Wang also proved a vanishing theorem for $L^{2}$ integrable harmonic 1-forms on $M$ in 8 :

Theorem 0.4 (Li-Wang). Let $M$ be an n-dimensional complete Riemannian manifold with $\lambda_{1}(M)>0$ and

$$
\operatorname{Ric}_{M} \geq-\frac{n}{n-1} \lambda_{1}(M)+\varepsilon
$$

for some $\varepsilon>0$. Then $H^{1}\left(L^{2}(M)\right)=0$.

In this article, we generalized the above theorem to manifolds satisfying a weighted Poincaré inequality:

Theorem 0.5. Let $M$ be an n-dimensional complete Riemannian manifold satisfying a weighted Poincaré inequality with a nonnegative weight function $\rho(x)$. Assume the Ricci curvature satisfies

$$
\operatorname{Ric}_{M}(x) \geq-\frac{n}{n-1} \rho(x)+\varepsilon,
$$

for some $\varepsilon>0$. Let $r(x, p)$ be the distance function from $x$ to some fixed point $p$. If $\rho(x)=O\left(r^{2-\alpha}(x, p)\right)$, for some $0<\alpha<2$, then $H^{1}\left(L^{2}(M)\right)=0$.

\section{Some Lemmas}

The following lemma is modified from 9 ] to suit our situation.

Lemma 1.1. Assume that

$$
\operatorname{Ric}_{M}(x) \geq-\frac{n-1}{n-2} l(x)
$$

for some function $l(x)$. If $f$ is a positive harmonic function on $M$, then

$$
|\nabla f|(x) \leq\left((n-1) \sup _{B_{\rho}(x, 1)} \sqrt{l(y)}+C_{1} \sup _{B_{\rho}(x, 1)} \sqrt{\rho(y)}\right) f(x),
$$

where $C_{1}$ is a constant only depending on $n$. In particular, if the lower bound of the Ricci curvature of $M$ satisfies

$$
\operatorname{Ric}_{M \backslash K}(x) \geq-\frac{n-1}{n-2} \rho(x)+\tilde{\varepsilon},
$$


where $K$ is a compact subdomain of $M$ and for some $\tilde{\varepsilon}>0$, then

$$
|\nabla f|(x) \leq C\left(\sup _{B_{\rho}(x, 1)} \sqrt{\rho}\right) f(x)
$$

where $C=C(n)$, provided that $B_{\rho}(x, 1) \cap K=\emptyset$.

Proof. Cheng and Yau's [2] (see also [8]) local gradient estimate for positive harmonic functions implies that for any $R>0$,

$$
|\nabla f|(x) \leq\left((n-1) \sup _{B(x, R)} \sqrt{l}+C R^{-1}\right) f(x),
$$

where $C=C(n)$. Consider the function $g(r)=r-\left(\sup _{B(x, r)} \sqrt{\rho}\right)^{-1}$. Since $g$ is negative when $r \rightarrow 0$ and $g \rightarrow+\infty$ as $r \rightarrow+\infty$, hence we can choose $R_{0}>0$ such that $g\left(R_{0}\right)=0$, that is, $R_{0}=\left(\sup _{B\left(x, R_{0}\right)} \sqrt{\rho}\right)^{-1}$. For any point $y \in B\left(x, R_{0}\right)$, let $\gamma$ be a minimizing geodesic (with respect to $d s_{M}^{2}$ ) joining $x, y$. Then

$$
\begin{aligned}
r_{\rho}(x, y) & \leq \int_{\gamma} \sqrt{\rho(\gamma(t))} d t \\
& \leq\left(\sup _{B\left(x, R_{0}\right)} \sqrt{(\rho(y))}\right) R_{0} \\
& \leq 1 ;
\end{aligned}
$$

hence $B\left(x, R_{0}\right) \subseteq B_{\rho}(x, 1)$. Combining with (1.2) by choosing $R=R_{0}$, the result follows.

The following version of the Bochner formula is well known and was first used by Yau [14.

Lemma 1.2 ([14] (see also [9])). Let $M^{n}$ be a complete Riemannian manifold of dimension $n \geq 2$. Assume that the Ricci curvature of $M$ satisfies the lower bound

$$
\operatorname{Ric}_{M}(x) \geq-(n-1) \tau(x),
$$

for some function $\tau$. Assume that $f$ is a nonconstant harmonic function on $M$. Then the function $h=|\nabla f|$ satisfies the following differential inequality:

$$
h \triangle h \geq-(n-1) \tau h^{2}+\frac{|\nabla h|^{2}}{n-1}
$$

in the weak sense. In addition, if we write $g=h^{\frac{n-2}{n-1}}$, the above inequality becomes

$$
\triangle g \geq-(n-2) \tau g
$$

Proof. For the sake of completeness, we outline the proof here. We choose a local orthonormal frame $\left\{e_{1}, e_{2}, \ldots, e_{n}\right\}$ such that $e_{1} f=|\nabla f|$ and $e_{\alpha} f=0$, for $\alpha=$ $2, \ldots, n$ at a point $x$,

$$
\begin{aligned}
h^{2} & =|\nabla f|^{2}, \\
h h_{j} & =\sum_{i=1}^{n} f_{i j} f_{i} .
\end{aligned}
$$


Hence

$$
\begin{aligned}
h_{j} & =f_{1 j}, \\
|\nabla h|^{2} & =\sum_{j=1}^{n} h_{j}^{2}=\sum_{j=1}^{n} f_{1 j}^{2},
\end{aligned}
$$

when evaluated at $x$. Using the fact that $f$ is harmonic and the Ricci formula, we compute

$$
\begin{aligned}
|\nabla h|^{2}+h \triangle h & =\sum_{j=1}^{n}\left(h_{j}^{2}+h h_{j j}\right) \\
& =\sum_{i, j=1}^{n}\left(f_{i j}^{2}+f_{i j j} f_{i}\right) \\
& =\sum_{i, j=1}^{n}\left(f_{i j}^{2}+f_{j j i} f_{i}+R_{i j} f_{i} f_{j}\right) \\
& =\sum_{i, j=1}^{n}\left(f_{i j}^{2}+R_{i j} f_{i} f_{j}\right) \\
& \geq-(n-1) \tau h^{2}+\sum_{i, j=1}^{n} f_{i j}^{2}
\end{aligned}
$$

and

$$
\begin{aligned}
\sum_{i, j=1}^{n} f_{i j}^{2} & =2 \sum_{\alpha=2}^{n} f_{1 \alpha}^{2}+f_{11}^{2}+\sum_{\beta=2}^{n} f_{\beta \beta}^{2} \\
& \geq 2 \sum_{\alpha=2}^{n} f_{1 \alpha}^{2}+f_{11}^{2}+\frac{1}{n-1}\left(\sum_{\beta=2}^{n} f_{\beta \beta}\right)^{2} \\
& =2 \sum_{\alpha=2}^{n} f_{1 \alpha}^{2}+\frac{n}{n-1} f_{11}^{2} \\
& \geq \frac{n}{n-1} \sum_{j=1}^{n} f_{1 j}^{2} .
\end{aligned}
$$

Combining the above inequality with (1.3), (1.4) and evaluating at $x$, the result follows.

A theory of Li and Tam [7] allows us to count the number of nonparabolic ends of $M$ by counting the dimension of $\mathcal{K}^{0}(M)$, a subspace of the space of all harmonic functions on $M$. We outline the construction of Li and Tam here. Assuming that $M$ has at least two nonparabolic ends, $E_{1}, E_{2}$, for $R>0$, we solve the following equation:

$$
\left\{\begin{array}{rlc}
\triangle f_{R}=0 & \text { in } & B(R), \\
f_{R}=1 & \text { on } & \partial B(R) \cap E_{1}, \\
f_{R}=0 & \text { on } & \partial B(R) \backslash E_{1} .
\end{array}\right.
$$

By passing to a convergent subsequence, the sequence $\left\{f_{R}\right\}$ converges to a nonconstant harmonic function $f_{1}$ with finite Dirichlet integral, satisfying $0 \leq f_{1} \leq 1$. 
Clearly for each nonparabolic end $E_{i}$, we can construct a corresponding $f_{i}$ by the above process. Let $\mathcal{K}^{0}(M)$ be the linear space containing all the $f_{i}$ 's constructed as above. By the construction, the number of nonparabolic ends of $M$ is given by the dimension of $\mathcal{K}^{0}(M)$. The following lemma of $\mathrm{Li}$ is useful in proving finiteness type theorems.

Lemma $1.3([5])$. Let $\mathcal{H}$ be a finite dimensional subspace of $L^{2}$ p-forms defined over a set $D \subseteq M^{n}$. If $V(D)$ denotes the volume of the set $D$, then there exists $\omega_{0} \in \mathcal{H}$ such that

$$
\operatorname{dim} \mathcal{H} \int_{D}\left|\omega_{0}\right|^{2} \leq V(D) \cdot \sup _{D}\left|\omega_{0}\right|^{2} \cdot \min \left\{\left(\begin{array}{l}
n \\
p
\end{array}\right), \operatorname{dim} \mathcal{H}\right\}
$$

\section{Finitely MANY ENDS}

Theorem 2.1. Let $M^{n}$ be a complete manifold with dimension $n \geq 3$. Assume that $M$ has property $\left(\mathcal{P}_{\rho}\right)$. Also assume that the Ricci curvature of $M$ satisfies the lower bound

$$
\operatorname{Ric}_{M \backslash K}(x) \geq-\frac{n-1}{n-2} \rho(x)+\tilde{\varepsilon}
$$

for some $\tilde{\varepsilon}>0$, compact set $K \subseteq M$. If $\rho$ satisfies the growth estimate

$$
\liminf _{R \rightarrow \infty} \frac{S(R)}{F(R)}=0
$$

where

$$
F(R)= \begin{cases}\exp \left(\frac{n-3}{n-2} R\right) & \text { for } n \geq 4, \\ R & \text { for } n=3\end{cases}
$$

then $M$ has only finitely many nonparabolic ends. If in addition $\liminf _{x \rightarrow \infty} \rho(x)>0$, then $M$ has only finitely many infinite volume ends.

Proof. By the discussion preceding Lemma 1.3, it is sufficient to estimate $\operatorname{dim} \mathcal{K}^{0}(M)$. We may assume that $M$ has at least two nonparabolic ends and hence there exists a nonconstant bounded harmonic function $f \in \mathcal{K}^{0}(M)$ with finite Dirichlet integral. By the maximal principle, we may assume that inf $f=0$ and $\sup f=1$ (see [7]). Using the assumption on the Ricci curvature and Lemma 1.2, we have

$$
\triangle|\nabla f| \geq-\frac{n-1}{n-2}|\nabla f|(\rho-\varepsilon)+\frac{\left.|\nabla| \nabla f\right|^{2}}{(n-1)|\nabla f|}
$$

in $M \backslash K$, where $\varepsilon=\frac{n-2}{n-1} \tilde{\varepsilon}$. Applying Lemma 1.2, the above equation becomes

$$
\triangle g+\rho g \geq \varepsilon g
$$

in $M \backslash K$, where $g=|\nabla f|^{\frac{n-2}{n-1}}$. Let $\phi \in C_{c}^{\infty}(M \backslash K)$ be a nonnegative smooth function with compact support in $M \backslash K$. Using the property $\left(\mathcal{P}_{\rho}\right)$ of $M$ and 
integration by parts, we have

$$
\begin{aligned}
\int_{M} \phi^{2} \rho g^{2} & \leq \int_{M}|\nabla(\phi g)|^{2} \\
& =\int_{M}|\nabla \phi|^{2} g^{2}+\int_{M} \phi^{2}|\nabla g|^{2}+2 \int_{M} \phi g\langle\nabla \phi, \nabla g\rangle \\
& =\int_{M}|\nabla \phi|^{2} g^{2}+\int_{M} \phi^{2}|\nabla g|^{2}+\frac{1}{2} \int_{M}\left\langle\nabla(\phi)^{2}, \nabla(g)^{2}\right\rangle \\
& =\int_{M}|\nabla \phi|^{2} g^{2}-\int_{M} \phi^{2} g \triangle g .
\end{aligned}
$$

Combining (2.1) and (2.2), we have

$$
\varepsilon \int_{M} \phi^{2} g^{2} \leq \int_{M}|\nabla \phi|^{2} g^{2}, \forall \phi \in C_{c}^{\infty}(M \backslash K) .
$$

Let $B(t)$ be the geodesic ball of radius $t$ with respect to some fixed point $p$. Since $K$ is compact, we may choose $R_{0}>0$ such that

$$
K \subseteq \bigcup_{x \in K} B_{\rho}(x, 1) \subseteq B\left(R_{0}-1\right)
$$

where we have used the facts that for any $\varepsilon>0, \rho+\varepsilon$ is strictly positive, the metric $d s_{\rho+\varepsilon}^{2}$ is complete and $B_{\rho}(x, 1) \subseteq B_{\rho+\varepsilon}(x, 1)$. Therefore $B_{\rho}(x, 1)$ is precompact. Let $R>0$ be such that $B\left(R_{0}\right) \subseteq B_{\rho}(R-1)$. The above inequality implies

$$
\varepsilon \int_{B_{\rho}(R) \backslash B\left(R_{0}-1\right)} \phi^{2} g^{2} \leq \int_{B_{\rho}(R) \backslash B\left(R_{0}-1\right)}|\nabla \phi|^{2} g^{2},
$$

for any $\phi \in C_{c}^{\infty}\left(B_{\rho}(R) \backslash B\left(R_{0}-1\right)\right)$. If we write $\phi=\psi \cdot \chi$, the right hand side of (2.3) becomes

$$
\begin{aligned}
\int_{B_{\rho}(R) \backslash B\left(R_{0}-1\right)}|\nabla \phi|^{2} g^{2} & \leq 2 \int_{B_{\rho}(R) \backslash B\left(R_{0}-1\right)}|\nabla \psi|^{2} \chi^{2} g^{2} \\
& +2 \int_{B_{\rho}(R) \backslash B\left(R_{0}-1\right)}|\nabla \chi|^{2} \psi^{2} g^{2} .
\end{aligned}
$$

Now we choose $\chi, \psi$ as in [9]:

$$
\chi(x)=\left\{\begin{array}{cl}
0 & \text { on } \mathcal{L}(0, \delta \bar{\varepsilon}) \cup \mathcal{L}(1-\delta \bar{\varepsilon}, 1), \\
(-\log \delta)^{-1}(\log f-\log (\delta \bar{\varepsilon})) & \text { on } \mathcal{L}(\delta \bar{\varepsilon}, \bar{\varepsilon}) \cap\left(M \backslash E_{1}\right), \\
(-\log \delta)^{-1}(\log (1-f)-\log (\delta \bar{\varepsilon})) & \text { on } \mathcal{L}(1-\bar{\varepsilon}, 1-\delta \bar{\varepsilon}) \cap E_{1}, \\
1 & \text { otherwise }
\end{array}\right.
$$

for some $0<\delta<1$ and $0<\bar{\varepsilon}<\frac{1}{2}$ to be determined later, where

$$
\begin{gathered}
\mathcal{L}(a, b)=\{x \in M: a<f(x)<b\}, \\
\psi(x)=\left\{\begin{array}{cl}
0 & \text { on } B\left(R_{0}-1\right), \\
1 & \text { on } B_{\rho}(R-1) \backslash B\left(R_{0}\right), \\
R-r_{\rho} & \text { on } B_{\rho}(R) \backslash B_{\rho}(R-1), \\
0 & \text { on } M \backslash B_{\rho}(R) .
\end{array}\right.
\end{gathered}
$$


The first term on the right hand side of (2.4) can be estimated by

$$
\begin{aligned}
2 \int_{B_{\rho}(R) \backslash B\left(R_{0}-1\right)}|\nabla \psi|^{2} \chi^{2} g^{2} & \leq 2 \int_{B_{\rho}(R) \backslash B_{\rho}(R-1)}|\nabla \psi|^{2} \chi^{2} g^{2} \\
& +2 \int_{B\left(R_{0}\right) \backslash B\left(R_{0}-1\right)}|\nabla \psi|^{2} \chi^{2} g^{2} .
\end{aligned}
$$

Now consider

$$
\begin{aligned}
& \int_{E_{1}}|\nabla \chi|^{2} \psi^{2} g^{2} \\
\leq & (\log \delta)^{-2} \int_{\mathcal{L}(1-\bar{\varepsilon}, 1-\delta \bar{\varepsilon}) \cap E_{1} \cap\left(B_{\rho}(R) \backslash B\left(R_{0}-1\right)\right)}|\nabla f|^{2+\frac{2(n-1)}{n-2}}(1-f)^{-2} \\
\leq & C S^{\frac{2(n-2)}{n-1}}(R+1)(\log \delta)^{-2} \int_{\mathcal{L}(1-\bar{\varepsilon}, 1-\delta \bar{\varepsilon}) \cap E_{1} \cap\left(B_{\rho}(R) \backslash B\left(R_{0}-1\right)\right)}|\nabla f|^{2}(1-f)^{\frac{2(n-2)}{n-1}-2} \\
\leq & C S^{\frac{2(n-2)}{n-1}}(R+1)(\log \delta)^{-2} \int_{\mathcal{L}(1-\bar{\varepsilon}, 1-\delta \bar{\varepsilon}) \cap E_{1} \cap B_{\rho}(R)}|\nabla f|^{2}(1-f)^{\frac{2(n-2)}{n-1}-2},
\end{aligned}
$$

where the second inequality follows from (1.1) by replacing $f$ by $1-f$ and the choice of $R_{0}$ so that $B_{\rho}(x, 1)$ does not intersect $K$ for any $x \in B_{\rho}(R) \backslash B\left(R_{0}-1\right)$. We can now follow the arguments as in 9 to yield

$$
\int_{B_{\rho}(R) \backslash B_{\rho}(R-1)}|\nabla \psi|^{2} \chi^{2} g^{2} \leq C S^{\frac{2(n-2)}{n-1}}(R)(\delta \bar{\varepsilon})^{\frac{-2}{n-1}} \exp (-2 R)
$$

and

$$
\begin{aligned}
& \int_{M}|\nabla \chi|^{2} \psi^{2} g^{2} \\
& \leq\left\{\begin{array}{cc}
C S^{\frac{2(n-2)}{n-1}}(R+1)(-\log \delta)^{-2}\left(1-\delta^{\frac{n-3}{n-1}}\right) \bar{\varepsilon}^{\frac{n-3}{n-1}}, & n \geq 4, \\
C S(R+1)(-\log \delta)^{-1}, & n=3,
\end{array}\right.
\end{aligned}
$$

where $C$ is a constant independent of $R$. Continuing to follow the arguments in 9], we choose

$$
\begin{gathered}
\delta=\frac{1}{2}, \bar{\varepsilon}=\exp (-2 R) \text { when } n \geq 4, \\
\delta=\bar{\varepsilon}=\exp (-R q(R)) \text { when } n=3,
\end{gathered}
$$

where $q(R)=\sqrt{\frac{S(R+1)}{R}}$. Combining with the growth assumption of $\rho$, we can now conclude as in 9 , that

$$
\begin{aligned}
\int_{M}|\nabla \chi|^{2} \psi^{2} g^{2} & \rightarrow 0 \quad \text { as } & R \rightarrow+\infty, \text { and } \\
\int_{B_{\rho}(R) \backslash B_{\rho}(R-1)}|\nabla \psi|^{2} \chi^{2} g^{2} \rightarrow 0 & \text { as } & R \rightarrow+\infty .
\end{aligned}
$$

Letting $R \rightarrow+\infty$ and combining the above results with (2.4) and (2.5), (2.3) becomes

$$
\varepsilon \int_{M \backslash B\left(R_{0}\right)} g^{2} \leq \tilde{C} \int_{B\left(R_{0}\right) \backslash B\left(R_{0}-1\right)} g^{2}
$$


where $\tilde{C}$ is a constant depending only on $n$, which implies

$$
\int_{B\left(2 R_{0}\right)} g^{2} \leq C \int_{B\left(R_{0}\right)} g^{2}
$$

where $C=C(\varepsilon, n)$. The function $g$ satisfies the differential inequality

$$
\triangle g \geq-\alpha g
$$

on $B\left(p, 2 R_{0}\right)$, where $\alpha=\inf _{B\left(p, 2 R_{0}\right)} R i c_{M}$, and the mean value inequality of Li and Tam [6] implies that

$$
\begin{aligned}
g^{2}(x) & \leq C_{1} \int_{B\left(x, R_{0}\right)} g^{2} \\
& \leq C_{1} \int_{B\left(p, 2 R_{0}\right)} g^{2}
\end{aligned}
$$

for any $x \in B\left(p, R_{0}\right)$, where $\nu=\inf _{x \in B\left(p, R_{0}\right)} V_{x}\left(R_{0}\right)$ and $C_{1}=C_{1}(n, \alpha, \nu)$. Combining the above equation with (2.7), we have

$$
\sup _{B\left(p, R_{0}\right)} g^{2} \leq C_{2} \int_{B\left(p, R_{0}\right)} g^{2},
$$

where $C_{2}=C_{2}(\varepsilon, n, \alpha, \nu)$. On the other hand, Schwarz's inequality implies that

$$
\int_{B\left(R_{0}\right)} g^{2} \leq\left(\int_{B\left(R_{0}\right)}|\nabla f|^{2}\right)^{\frac{n-2}{n-1}} V_{p}\left(R_{0}\right)^{\frac{1}{n-1}} .
$$

Therefore we have

$$
\sup _{B\left(R_{0}\right)}|\nabla f|^{2} \leq C_{3} \int_{B\left(R_{0}\right)}|\nabla f|^{2},
$$

where $C_{3}=C_{3}\left(\varepsilon, n, \alpha, \nu, R_{0}\right)$ is a constant independent of $f \in \mathcal{K}^{0}(M)$. By unique continuation,

$$
\int_{B\left(R_{0}\right)}|\nabla f|^{2} \neq 0
$$

provided that $f$ is not a constant function. Therefore,

$$
\int_{B\left(R_{0}\right)}\langle\nabla f, \nabla g\rangle
$$

defines a nondegenerate bilinear form on the space of 1-forms

$$
\mathcal{K}=\left\{d f: f \in \mathcal{K}^{0}(M)\right\} .
$$

Lemma 1.3 asserts that there exists $f_{0} \in \mathcal{K}^{0}(M)$ such that

$$
\operatorname{dim} \mathcal{K} \int_{B\left(R_{0}\right)}\left|d f_{0}\right|^{2} \leq n V_{p}\left(R_{0}\right) \sup _{B\left(R_{0}\right)}\left|d f_{0}\right|^{2} .
$$

Combining the above with (2.8) implies

$$
\operatorname{dim} \mathcal{K}^{0}(M)=\operatorname{dim} \mathcal{K}+1 \leq C_{4}
$$

for some fixed constant $C_{4}=C_{4}\left(C_{3}, V_{p}\left(R_{0}\right)\right)$, which completes the proof. The second part of the theorem follows from $[9$; an end is nonparabolic if and only if it has infinite volume, provided that $\liminf _{x \rightarrow \infty} \rho>0$. 
Remark 2.2. We would like to point out that the growth condition is not too restrictive. It is satisfied if the weight function $\rho$ does not grow too fast, for instance, if $\rho$ has only polynomial growth $(n \geq 4)$.

\section{VANISHING THEOREMS}

In this section we study the space of $L^{2}$ integrable harmonic 1-forms, $H^{1}\left(L^{2}(M)\right)$. If $f$ is a harmonic function with finite Dirichlet integral, then the exterior derivative of $f, d f$ is an $L^{2}$ integrable harmonic 1-form. By the theory of Li and Tam 7] (see also [8]), we have

$$
\begin{aligned}
\operatorname{dim} H^{1}\left(L^{2}(M)\right)+1 & \geq \operatorname{dim} \mathcal{K}^{0}(M) \\
& \geq \text { number of nonparabolic ends of } M .
\end{aligned}
$$

If we further assume that $\lambda_{1}(M)>0$, then

$$
\operatorname{dim} H^{1}\left(L^{2}(M)\right)+1 \geq \text { number of infinite volume ends of } M .
$$

Therefore, an estimate on $\operatorname{dim} H^{1}\left(L^{2}(M)\right)$ is, in general, a stronger estimate than an estimate on the number of nonparabolic ends (infinite volume ends if, in addition, $\lambda_{1}(M)>0$ ). It is known that if $\omega$ is an $L^{2}$ harmonic 1-form, then it is both closed and co-closed. In particular, $h=|\omega|$ satisfies a Bochner type formula

$$
\triangle h \geq \frac{R i c_{M}(\omega, \omega)}{h}+\frac{|\nabla h|^{2}}{(n-1) h} .
$$

We start by proving an estimate for functions that satisfy the above Bochner type formula. We believe the estimate is of independent interest and will be useful in many other situations.

Lemma 3.1. Let $b>-1$. Assume that $h$ is a nonnegative function and satisfies the differential inequality

$$
h \triangle h \geq-a h^{2}+b|\nabla h|^{2}
$$

in the weak sense, for some constant a. For any $\varepsilon>0$, we have the following estimate:

$$
(b(1-\varepsilon)+1) \int_{M}|\nabla(\phi h)|^{2} \leq\left(b\left(\frac{1}{\varepsilon}-1\right)+1\right) \int_{M} h^{2}|\nabla \phi|^{2}+a \int_{M} \phi^{2} h^{2},
$$

for any compactly supported smooth function $\phi \in C_{c}^{\infty}(M)$. In addition, if

$$
\int_{B_{p}(R)} h^{2}=o\left(R^{2}\right)
$$

then

$$
\int_{M}|\nabla h|^{2} \leq \frac{a}{b+1} \int_{M} h^{2} .
$$

In particular, $h$ has finite Dirichlet integral if $h \in L^{2}(M)$. 
Proof. Let $\phi \in C_{c}^{\infty}(M)$ be a smooth function with compact support. Integration by parts implies

$$
\begin{aligned}
\int_{M} \phi^{2} h \triangle h & =-\int_{M}\left\langle\nabla\left(\phi^{2} h\right), \nabla h\right\rangle \\
& =\int_{M}\langle h \nabla \phi+\nabla(\phi h), h \nabla \phi-\nabla(\phi h)\rangle \\
& =\int_{M} h^{2}|\nabla \phi|^{2}-\int_{M}|\nabla(\phi h)|^{2} .
\end{aligned}
$$

By the differential inequality satisfied by $h$, we have

$$
\int_{M} \phi^{2} h \triangle h \geq-a \int_{M} \phi^{2} h^{2}+b \int_{M} \phi^{2}|\nabla h|^{2} .
$$

Combining the above inequality with (3.1) gives

$$
\int_{M} h^{2}|\nabla \phi|^{2}+a \int_{M} \phi^{2} h^{2} \geq b \int_{M} \phi^{2}|\nabla h|^{2}+\int_{M}|\nabla(\phi h)|^{2} .
$$

On the other hand, the Schwarz inequality implies

$$
\begin{aligned}
\int_{M} \phi^{2}|\nabla h|^{2} & =\int_{M}\langle\nabla(\phi h)-h \nabla \phi, \nabla(\phi h)-h \nabla \phi\rangle \\
& =\int_{M}\left(|\nabla(\phi h)|^{2}-2\langle\nabla(\phi h), h \nabla \phi\rangle+h^{2}|\nabla \phi|^{2}\right) \\
& \geq(1-\varepsilon) \int_{M}|\nabla(\phi h)|^{2}+\left(1-\frac{1}{\varepsilon}\right) \int_{M} h^{2}|\nabla \phi|^{2},
\end{aligned}
$$

for any $\varepsilon>0$. Combining the above with (3.2) gives the first result of the lemma. For the second part, we choose

$$
\phi= \begin{cases}1 & \text { on } B(R) \\ 0 & \text { on } M \backslash B(2 R)\end{cases}
$$

such that $|\nabla \phi| \leq C / R$ on $B(2 R) \backslash B(R)$. The estimate of the lemma implies

$$
(b(1-\varepsilon)+1) \int_{B(R)}|\nabla h|^{2} \leq\left(b\left(\frac{1}{\varepsilon}-1\right)+1\right) R^{-2} \int_{B(2 R) \backslash B(R)} h^{2}+a \int_{M} h^{2} .
$$

Using $\int_{B_{p}(R)} h^{2}=o\left(R^{2}\right)$, and letting $R \rightarrow+\infty, \varepsilon \rightarrow 0$, the second part of the lemma is achieved.

Remark 3.2. By the proof of the above lemma, the above conclusions are still valid if we assume that $a=a(x)$ is a function of $x$, in the following forms:

$$
(b(1-\varepsilon)+1) \int_{M}|\nabla(\phi h)|^{2} \leq\left(b\left(\frac{1}{\varepsilon}-1\right)+1\right) \int_{M} h^{2}|\nabla \phi|^{2}+\int_{M} a(x) \phi^{2} h^{2}
$$

and

$$
\int_{M}|\nabla h|^{2} \leq \frac{1}{b+1} \int_{M} a(x) h^{2} .
$$

The following theorem is an immediate application of the above lemma. 
Corollary 3.3. Let $h$ be a function satisfying the assumptions in Lemma 3.1. Assume that $\lambda_{1}(M)>0$ and the Ricci curvature of $M$ satisfies

$$
\operatorname{Ric}_{M} \geq-(b+1) \lambda_{1}(M)+\delta,
$$

for some $\delta>0$. If

$$
\int_{B_{p}(R)} h^{2}=o\left(R^{2}\right)
$$

then $h \equiv 0$. In particular, we recover a theorem of Li and Wang in [8]: Let $M^{n}$ be a complete noncompact $n$-dimensional Riemannian manifold with $\lambda_{1}(M)>0$, and the Ricci curvature satisfies

$$
\operatorname{Ric}_{M} \geq-\frac{n}{n-1} \lambda_{1}(M)+\delta
$$

for some $\delta>0$. Then $H^{1}\left(L^{2}(M)\right)=0$.

Proof. Combining Lemma 3.1 with the variational principle of $\lambda_{1}(M)$, we have

$$
\begin{aligned}
(b(1-\varepsilon)+1) \lambda_{1}(M) \int_{M} \phi^{2} h^{2} & \leq(b(1-\varepsilon)+1) \int_{M}|(\nabla \phi h)|^{2} \\
& \leq a \int_{M} \phi^{2} h^{2}+\left(b\left(\frac{1}{\varepsilon}-1\right)+1\right) \int_{M} h^{2}|\nabla \phi|^{2},
\end{aligned}
$$

for any $\varepsilon>0$ and any compactly supported smooth function $\phi \in C_{c}^{\infty}(M)$. The above inequality implies

$$
\delta \int_{M} \phi^{2} h^{2} \leq b \varepsilon \lambda_{1}(M) \int_{M} \phi^{2} h^{2}+\left(b\left(\frac{1}{\varepsilon}-1\right)+1\right) \int_{M} h^{2}|\nabla \phi|^{2} .
$$

Let

$$
\phi= \begin{cases}1 & \text { on } B(R), \\ 0 & \text { on } M \backslash B(2 R),\end{cases}
$$

such that $|\nabla \phi|^{2} \leq C / R^{2}$ on $B(2 R) \backslash B(R)$. The above inequality becomes

$$
\delta \int_{B(R)} h^{2} \leq b \varepsilon \lambda_{1}(M) \int_{B(2 R)} h^{2}+R^{-2}\left(b\left(\frac{1}{\varepsilon}-1\right)+1\right) \int_{B(2 R) \backslash B(R)} h^{2} .
$$

Combining the above inequality with the growth assumption on $h$, we let $R \rightarrow+\infty$, then take $\varepsilon \rightarrow 0$ and conclude that $\int_{M} h^{2} \leq 0$. Thus $h \equiv 0$. For the second part, let $\omega \in H^{1}\left(L^{2}(M)\right)$ and $h=|\omega| \in L^{2}(M)$. Then it is well known that $h$ satisfies the differential inequality

$$
h \triangle h \geq-a h^{2}+b|\nabla h|^{2}
$$

in the weak sense with $a=(b+1) \lambda_{1}(M)-\delta$ and $b=1 /(n-1)$ for any Riemannian manifold of dimension $n$.

Theorem 3.4. Let $M^{n}$ be a complete noncompact manifold of dimension $n$ satisfying the weighted Poincaré inequality with a nonnegative weight function $\rho(x)$. Assume the Ricci curvature satisfies

$$
\operatorname{Ric}_{M}(x) \geq-\frac{n}{n-1} \rho(x)+\delta,
$$

for some $\delta>0$. If $\rho(x)$ is of subquadratic growth of the distance function, that is,

$$
\rho(x)=O\left(r_{p}^{2-\alpha}(x)\right)
$$


where $r_{p}(x)$ is the distance function from $x$ to some fixed point $p$, for some $0<\alpha<$ 2 , then $H^{1}\left(L^{2}(M)\right)=0$.

Proof. Let $\omega \in H^{1}\left(L^{2}(M)\right)$ and $h=|\omega| \in L^{2}(M)$. Applying Lemma 3.1 with $b=\frac{1}{n-1}, a=(b+1) \rho-\delta$ and using the weighted Poincaré inequality, we have

$$
\begin{aligned}
(b(1-\varepsilon)+1) \int_{M} \rho \phi^{2} h^{2} & \leq(b(1-\varepsilon)+1) \int_{M}|(\nabla \phi h)|^{2} \\
& \leq \int_{M} a(x) \phi^{2} h^{2}+\left(b\left(\frac{1}{\varepsilon}-1\right)+1\right) \int_{M} h^{2}|\nabla \phi|^{2} .
\end{aligned}
$$

Thus

$$
\delta \int_{M} \phi^{2} h^{2} \leq\left((1-b)+b \varepsilon^{-1}\right) \int_{M} h^{2}|\nabla \phi|^{2}+b \varepsilon \int_{M} \rho \phi^{2} h^{2},
$$

for any $\varepsilon>0$ and any compactly supported smooth function $\phi \in C_{c}^{\infty}(M)$. Let $\varepsilon=R^{\alpha / 2-2}$ and

$$
\phi= \begin{cases}1 & \text { on } B(R) \\ 0 & \text { on } M \backslash B(2 R)\end{cases}
$$

such that $|\nabla \phi|^{2} \leq C / R^{2}$ on $B(2 R) \backslash B(R)$. The above inequality becomes

$$
\delta \int_{B(R)} h^{2} \leq b R^{-\alpha / 2} \int_{B(2 R)} h^{2}+\left((1-b) R^{-2}+b R^{-\alpha / 2}\right) \int_{B(2 R)} h^{2} .
$$

Combining the above inequality with the assumption $h \in L^{2}(M)$ and letting $R \rightarrow$ $+\infty$, we conclude that $\int_{M} h^{2} \leq 0$ and thus $h \equiv 0$.

Theorem 3.5. Let $M^{n}$ be a complete noncompact manifold of dimension $n$ satisfying the weighted Poincaré inequality with weight function $\rho>0$. Assume the Ricci curvature satisfies $\operatorname{Ric}_{M} \geq-\left(\frac{n}{n-1}-\delta\right) \rho$, for some $\delta>0$, and $\rho=O\left(r_{p}^{2-\alpha}(x)\right)$ for some $0<\alpha<2$. Then $H^{1}\left(L^{2}(M)\right)=0$.

Proof. Let $\omega \in H^{1}\left(L^{2}(M)\right)$ and $h=|\omega| \in L^{2}(M)$. Applying Lemma 3.1 with $b=\frac{1}{n-1}, a=(b+1-\delta) \rho$, and weighted Poincaré inequality, we have

$$
\begin{aligned}
(b(1-\varepsilon)+1) \int_{M} \rho \phi^{2} h^{2} & \leq(b(1-\varepsilon)+1) \int_{M}|(\nabla \phi h)|^{2} \\
& \leq a \int_{M} \phi^{2} h^{2}+\left(b\left(\frac{1}{\varepsilon}-1\right)+1\right) \int_{M} h^{2}|\nabla \phi|^{2} .
\end{aligned}
$$

Thus

$$
\delta \int_{M} \rho \phi^{2} h^{2} \leq b \varepsilon \int_{M} \rho \phi^{2} h^{2}+\left(b \varepsilon^{-1}-b+1\right) \int_{M} h^{2}|\nabla \phi|^{2} .
$$

If we choose

$$
\phi= \begin{cases}1 & \text { on } B(R), \\ 0 & \text { on } M \backslash B(2 R),\end{cases}
$$

using $\rho(x)=O\left(r^{2-\alpha}(x, p)\right)$ and letting $\varepsilon=R^{\alpha / 2-2}$, the above inequality implies

$$
\delta \int_{B(R)} \rho h^{2} \leq C R^{-\alpha / 2} \int_{B(2 R)} h^{2}+\left(b R^{-\alpha / 2}+(1-b) R^{-2}\right) \int_{B(2 R)} h^{2},
$$


for some constant $C$. Using $h \in L^{2}(M)$ and letting $R \rightarrow+\infty$, we conclude that $\int_{M} \rho h^{2}=0$. By Lemma 3.1, we have

$$
\begin{aligned}
\int_{M}|\nabla h|^{2} & \leq\left(1-\frac{\delta}{b+1}\right) \int_{M} \rho h^{2} \\
& =0 .
\end{aligned}
$$

Therefore $|\nabla h| \equiv 0$. Hence $h \equiv c \in L^{2}(M)$ for some constant $c$. Since $M$ is nonparabolic, it must have infinite volume and thus $h \equiv 0$.

\section{MANifolds With PARALLEL FORMS}

The following theorem in 3 will allow us to obtain some improved vanishing theorems for manifolds with parallel forms when combining with the result in the previous section.

Theorem 4.1 ([3]). Let $M^{n}$ be an n-dimensional complete Riemannian manifold with a parallel $p$-form $\omega$. Assume that $\alpha$ is an $L^{2}$ harmonic 1 -form, that is, $\triangle \alpha=0$ and

$$
\int_{M}|\alpha|^{2}<+\infty
$$

Then $\alpha$ satisfies

$$
d *(\alpha \wedge \omega)=0 .
$$

Proof. For the sake of completeness, we outline the proof here. First, we claim that

$$
* d *(\alpha \wedge \omega)=(-1)^{n-1} d *(\alpha \wedge * \omega) .
$$

For any $x \in M$, we choose a local orthonormal frame $\left\{e_{i}\right\}_{i=1}^{n}$ such that $\nabla_{e_{i}} e_{j}(x)=$ 0 . Let $\left\{\theta^{i}\right\}_{i=1}^{n}$ be the coframe. For any $p$-form $\omega$, we have

$$
d \omega=\varepsilon\left(\theta^{i}\right) \nabla_{e_{i}} \omega
$$

at $x$ and $\omega$ is parallel if and only if $\nabla_{e_{i}} \omega=0, \forall i$. Let $\alpha=\sum_{i=1}^{n} a_{i} \theta^{i}$, and hence $\bar{\alpha}=\sum_{i=1}^{n} a_{i} e_{i}$ is the dual of $\alpha$. We use the notation $d \alpha=\sum_{i, j=1}^{n} a_{i, j} \theta^{j} \wedge \theta^{i}$, where $a_{i, j}=\nabla_{e_{j}} a_{i}$. Since $\alpha$ is $L^{2}$ harmonic, it is both closed and co-closed, which are equivalent to the conditions that $a_{i, j}=a_{j, i}$ and $\sum_{i=1}^{n} a_{i, i}=0$. The following calculations are all evaluated at $x$ :

$$
\begin{aligned}
d *(\alpha \wedge * \omega) & =d * \varepsilon(\alpha) * \omega \\
& =(-1)^{(p-1)(n-p)} d[l(\bar{\alpha}) \omega] \\
& =(-1)^{(p-1)(n-p)} \sum_{i=1}^{n} \varepsilon\left(\theta^{i}\right) \nabla_{e_{i}}(l(\bar{\alpha}) \omega) \\
& =(-1)^{(p-1)(n-p)} \sum_{i, j=1}^{n} \varepsilon\left(\theta^{i}\right) a_{j, i}\left(l\left(e_{j}\right) \omega\right),
\end{aligned}
$$


where the third equality follows from $\nabla_{e_{i}} e_{j}(x)=0$ and the last equality follows from $\nabla \omega=0$. On the other hand,

$$
\begin{aligned}
* d *(\alpha \wedge \omega) & =* d * \varepsilon(\alpha) \omega \\
& =* \sum_{i=1}^{n} \varepsilon\left(\theta^{i}\right) \nabla_{e_{i}}\left(* \varepsilon\left(\sum_{j=1}^{n} a_{j} \theta^{j}\right) \omega\right) \\
& =*\left(\sum_{i, j=1}^{n} a_{j, i} \varepsilon\left(\theta^{i}\right) *\left(\varepsilon\left(\theta^{j}\right) \omega\right)\right) \\
& =(-1)^{p(n-p-1)} \sum_{i, j=1}^{n} a_{i, j} l\left(e_{i}\right) \varepsilon\left(\theta^{j}\right) \omega \\
& =(-1)^{p(n-p-1)}\left(\sum_{i=1}^{n} a_{i, i} l\left(e_{i}\right) \varepsilon\left(\theta^{i}\right) \omega+\sum_{i \neq j}^{n} a_{i, j} l\left(e_{i}\right) \varepsilon\left(\theta^{j}\right) \omega\right) \\
& =(-1)^{p(n-p-1)}\left(\sum_{i=1}^{n} a_{i, i}\left[\omega-\varepsilon\left(\theta^{i}\right) l\left(e_{i}\right) \omega\right]\right. \\
& \left.-\sum_{i \neq j}^{n} a_{i, j} \varepsilon\left(\theta^{j}\right) l\left(e_{i}\right) \omega\right) \\
& =(-1)^{p(n-p-1)+1} \sum_{i, j=1}^{n} a_{i, j} \varepsilon\left(\theta^{i}\right)\left(l\left(e_{j}\right) \omega\right),
\end{aligned}
$$

where the last equality follows from $a_{i, j}=a_{j, i}$ and $\sum_{i=1}^{n} a_{i, i}=0$. (4.1) now follows from (4.2) and (4.3). Let

$$
\phi(x)=\left\{\begin{array}{llc}
1 & \text { on } & B_{p}(R) \\
0 & \text { on } & M \backslash B_{p}(2 R)
\end{array}\right.
$$

such that $|\nabla \phi| \leq C_{1} R^{-1}$. Consider

$$
\begin{aligned}
\int_{M} \phi^{2}|d *(\alpha \wedge \omega)|^{2} & =\left|\int_{M} \phi^{2} d *(\alpha \wedge \omega) \wedge * d *(\alpha \wedge \omega)\right| \\
& =\left|\int_{M} \phi^{2} d *(\alpha \wedge \omega) \wedge d *(\alpha \wedge * \omega)\right| \\
& =\left|\int_{M} d \phi^{2} \wedge *(\alpha \wedge \omega) \wedge d *(\alpha \wedge * \omega)\right| \\
& \leq 2\left(\int_{M}|d \phi|^{2}|*(\alpha \wedge \omega)|\right)^{1 / 2}\left(\int_{M} \phi^{2}|d *(\alpha \wedge * \omega)|^{2}\right)^{1 / 2} \\
& =2\left(\int_{M}|d \phi|^{2}|*(\alpha \wedge \omega)|\right)^{1 / 2}\left(\int_{M} \phi^{2}|d *(\alpha \wedge \omega)|^{2}\right)^{1 / 2}
\end{aligned}
$$

where the second and the last equality follow from (4.1), the third equality follows from integration by parts and the fact that $d^{2}=0 . \omega$ is parallel implies

$$
|*(\alpha \wedge \omega)| \leq C_{2}|\alpha|
$$


for some constant $C_{2}$. Combining the above with (4.4), we have

$$
\int_{B_{p}(R)}|d *(\alpha \wedge \omega)|^{2} \leq C_{3} R^{-2} \int_{B_{p}(2 R)}|\alpha|^{2} .
$$

Letting $R \rightarrow+\infty$, the result follows from the assumption that $\alpha$ is $L^{2}$ integrable.

Theorem 4.2. Let $M^{2 n}$ be a real $2 n$-dimensional Kähler manifold with $\lambda_{1}(M)>0$. Assume the Ricci curvature of $M$ satisfies

$$
\operatorname{Ric}_{M} \geq-2 \lambda_{1}(M)+\delta
$$

for some $\delta>0$. Then $H^{1}\left(L^{2}(M)\right)=0$.

Proof. Let $\omega \in H^{1}\left(L^{2}(M)\right)$ and $h=|\omega|$. We claim that $h$ satisfies the Bochner formula of the following form:

$$
\triangle h \geq \frac{\operatorname{Ric}_{M}(\omega, \omega)}{h}+\frac{|\nabla h|^{2}}{h} .
$$

Applying Corollary 3.3 with $b=1$, the result follows. To prove the claim, we let $\left\{e_{i}\right\}_{i=1}^{2 n}=\left\{\bar{e}_{1}, \ldots, \bar{e}_{n}, I \bar{e}_{1}, \ldots, I \bar{e}_{n}\right\}$ be a local orthonormal frame, where $I$ is the complex structure and let $\left\{\theta^{i}\right\}_{i=1}^{2 n}=\left\{\bar{\theta}^{1}, \ldots, \bar{\theta}^{n}, I \bar{\theta}^{1}, \ldots, I \bar{\theta}^{n}\right\}$ be the orthonormal coframe. The Kähler form, satisfying $\Omega(X, Y)=g(X, I Y)$, is then given by

$$
\begin{aligned}
\Omega & =-\sum_{i=1}^{n} \bar{\theta}^{i} \wedge I \bar{\theta}^{i} \\
& =-\sum_{i=1}^{n} \theta^{i} \wedge \theta^{n+i}
\end{aligned}
$$

With the above notation, we can write $\omega=\sum_{i=1}^{2 n} a_{i} \theta^{i}$. By Theorem 4.1,

$$
d *(\omega \wedge \Omega)=0
$$

which is equivalent to, by (4.3),

$$
\sum_{i, j=1}^{2 n} a_{i j} \theta^{i} \wedge l\left(e_{j}\right) \Omega=0
$$

where we have used the notation $a_{i j}=a_{i, j}$. Since

$$
l\left(e_{j}\right) \Omega=\left\{\begin{array}{cc}
-\theta^{j+n} & 1 \leq j \leq n, \\
\theta^{j-n} & n+1 \leq j \leq 2 n
\end{array}\right.
$$

hence (4.5) becomes

$$
\sum_{i=1}^{2 n}\left(-\sum_{j=1}^{n} a_{i j} \theta^{i} \wedge \theta^{j+n}+\sum_{j=1}^{n} a_{i, j+n} \theta^{i} \wedge \theta^{j}\right)=0 .
$$

The coefficient of $\theta^{i} \wedge \theta^{i+n}$ in the above equation is zero, and thus we conclude that

$$
a_{i i}+a_{i+n, i+n}=0
$$


for any $1 \leq i \leq n$. Now we go back to studying the form $\omega$. Let $\left\{e_{i}\right\}_{i=1}^{2 n}$ be as described above with $e_{1}$ such that $\omega\left(e_{1}\right)=|\omega|$ and $\omega\left(e_{j}\right)=0$ for any $j \neq 1$ at a fixed point $p$. Then

$$
\begin{aligned}
|\nabla \theta|^{2} & =\sum_{i, j=1}^{2 n} a_{i j}^{2} \\
& \geq a_{11}^{2}+a_{n+1, n+1}^{2}+2 \sum_{j=2}^{2 n} a_{1 j}^{2} \\
& =2\left(a_{11}^{2}+\sum_{j=2}^{2 n} a_{1 j}^{2}\right) \\
& =2|\nabla h|^{2}
\end{aligned}
$$

at $p$, where the third equality follows from (4.6). Combining the above inequality with the Bochner formula gives us

$$
\begin{aligned}
\frac{1}{2} \triangle\left(h^{2}\right) & =\operatorname{Ric}(\omega, \omega)+|\nabla \theta|^{2} \\
& \geq 2|\nabla h|^{2}+\operatorname{Ric}(\omega, \omega) .
\end{aligned}
$$

Hence

$$
\triangle h \geq \frac{\operatorname{Ric}_{M}(\omega, \omega)}{h}+\frac{|\nabla h|^{2}}{h},
$$

and the claim is justified.

Theorem 4.3. Let $M^{4 n}$ be a $4 n$-dimensional quaternionic Kähler manifold. Assume that $\lambda_{1}(M)>0$ and that the Ricci curvature of $M$ satisfies

$$
\operatorname{Ric}_{M} \geq-\frac{4}{3} \lambda_{1}(M)+\delta
$$

for some $\delta>0$. Then $H^{1}\left(L^{2}(M)\right)=0$.

Proof. We follow the notation in 11]. $M$ has a rank 3 vector bundle $V \subseteq \operatorname{End}(T M)$ satisfying

(1) In a local coordinate neighborhood, there exists a local basis $\{I, J, K\}$ of $V$ such that

$$
\begin{array}{r}
I^{2}=J^{2}=K^{2}=-1, \\
I J=-J I=K, \\
J K=-K J=I, \\
K I=-I K=J
\end{array}
$$

and

$$
g(X, Y)=g(I X, I Y)=g(J X, J Y)=g(K X, K Y),
$$

for any $X, Y \in T M$.

(2) If $\phi \in \Gamma(V)$, then $\nabla_{X} \phi \in \Gamma(V)$ for any $X \in T M$. 
We define the following two-forms:

$$
\begin{aligned}
& \omega_{1}(X, Y)=g(X, I Y), \\
& \omega_{2}(X, Y)=g(X, J Y), \\
& \omega_{3}(X, Y)=g(X, K Y) .
\end{aligned}
$$

The parallel 4-form of $M$ is then given by

$$
\Omega=\omega_{1} \wedge \omega_{1}+\omega_{2} \wedge \omega_{2}+\omega_{3} \wedge \omega_{3} .
$$

Let

$$
\left\{e_{i}\right\}_{i=1}^{4 n}=\left\{\bar{e}_{1}, \ldots, \bar{e}_{n}, I \bar{e}_{1}, \ldots, I \bar{e}_{n}, J \bar{e}_{1}, \ldots, J \bar{e}_{n}, K \bar{e}_{1}, \ldots, K \bar{e}_{n}\right\}
$$

be a local orthonormal frame and let

$$
\left\{\omega^{i}\right\}_{i=1}^{4 n}=\left\{\bar{\theta}^{1}, \ldots, \bar{\theta}^{n}, I \bar{\theta}^{1}, \ldots, I \bar{\theta}^{n}, J \bar{\theta}^{1}, \ldots, J \bar{\theta}^{n}, K \bar{\theta}^{1}, \ldots, K \bar{\theta}^{n}\right\}
$$

be the orthonormal coframe. Let $\omega=\sum_{i=1}^{4 n} a_{i} \omega^{i} \in H^{1}\left(L^{2}(M)\right)$. Using the above formula of $\Omega$ and calculating as in Theorem 4.2 (or see [11]), we have

$$
a_{i i}+a_{i+n, i+n}+a_{i+2 n, i+2 n}+a_{i+3 n, i+3 n}=0,
$$

for any $1 \leq i \leq n$. We now proceed as in Theorem 4.2. Let $h=|\omega|$. It is not difficult to see that $h$ satisfies the Bochner formula of the following form:

$$
\triangle h \geq \frac{\operatorname{Ric}_{M}(\omega, \omega)}{h}+\frac{1}{3} \frac{|\nabla h|^{2}}{h} .
$$

Applying Corollary 3.3 with $b=\frac{1}{3}$, the result follows. Indeed, let $\left\{e_{i}\right\}_{i=1}^{4 n}$ be as described above with $e_{1}$ such that $\omega\left(e_{1}\right)=|\omega|$ and $\omega\left(e_{j}\right)=0$ for any $j \neq 1$ at a point $p$. We compute

$$
\begin{aligned}
|\nabla \theta|^{2} & =\sum_{i, j=1}^{4 n} a_{i j}^{2} \\
& \geq a_{11}^{2}+a_{1+n, 1+n}^{2}+a_{1+2 n, 1+2 n}^{2}+a_{1+3 n, 1+3 n}^{2}+2 \sum_{j=2}^{2 n} a_{1 j}^{2} \\
& \geq a_{11}^{2}+\frac{1}{3}\left(a_{1+n, 1+n}+a_{1+2 n, 1+2 n}+a_{1+3 n, 1+3 n}\right)^{2}+2 \sum_{j=2}^{2 n} a_{1 j}^{2} \\
& =\frac{4}{3} a_{11}^{2}+2\left(\sum_{j=2}^{2 n} a_{1 j}^{2}\right) \\
& =\frac{4}{3}\left(a_{11}^{2}+\sum_{j=2}^{2 n} a_{1 j}^{2}\right) \\
& =\frac{4}{3}|\nabla h|^{2}
\end{aligned}
$$

at $p$, where the third inequality and the fourth equality follow from Schwarz's inequality and from (4.7), respectively. Combining the above inequality with the 
Bochner formula gives us

Hence

$$
\begin{aligned}
\frac{1}{2} \triangle\left(h^{2}\right) & =\operatorname{Ric}(\omega, \omega)+|\nabla \theta|^{2} \\
& \geq \frac{4}{3}|\nabla h|^{2}+\operatorname{Ric}(\omega, \omega) .
\end{aligned}
$$

$$
\triangle h \geq \frac{\operatorname{Ric}_{M}(\omega, \omega)}{h}+\frac{1}{3} \frac{|\nabla h|^{2}}{h} .
$$

\section{ACKNOWLEDGEMEnT}

The author would like to express his deepest gratitude to his advisor, Professor Peter Li, for his continuous support and constant encouragement. The author would also like to thank Professor Jiaping Wang for many suggestions in this work.

\section{REFERENCES}

1. M. Cai and G. J. Galloway, Boundaries of zero scalar curvature in the AdS/CFT correspondence, Adv. Theor. Math. Phys. 3 (1999), 1769-1783. MR1812136 (2002k:53080)

2. S. Y. Cheng and S. T. Yau, Differential equations on Riemannian manifolds and their geometric applications, Comm. Pure Appl. Math. 28 (1975), 333-354. MR0385749 (52:6608)

3. K. H. Lam, Spectrum of the Laplacian on manifolds with Spin(9) holonomy, Preprint, arXiv:0711.1428.

4. J. Lee, The spectrum of an asymptotic hyperbolic Einstein manifold, Comm. Anal. Geom. 3 (1995), 253-271. MR1362652 (96h:58176)

5. P. Li, On the Sobolev constant and the p-spectrum of a compact Riemannian manifold, Ann. Scient. Éc. Norm. Sup. 4e série, t. 13 (1980), 451-469. MR608289 (82h:58054)

6. P. Li and L. F. Tam, The heat equation and harmonic maps of complete manifolds, Invent. Math. 105 (1991), 1-46. MR.1109619 (93e:58039)

7. _ Harmonic functions and the structure of complete manifolds, J. Diff. Geom. 35 (1992), 359-383. MR:1158340 (93b:53033)

8. P. Li and J. Wang, Complete manifolds with positive spectrum, J. Diff. Geom. 58 (2001), 501-534. MR1906784 (2003e:58046)

9. Weighted Poincaré inequality and rigidity of complete manifolds, Ann. Scient. Éc. Norm. Sup., 4e série, t. 39 (2006), 921-982. MR2316978 (2008d:53053)

10. R. Mazzeo, The Hodge cohomology of a conformally compact metric, J. Diff. Geom. 28 (1988), 309-339. MR961517 (89i:58005)

11. S. Kong, P. Li, and D. Zhou, Spectrum of the Laplacian on quaternionic Kähler manifolds, J. Diff. Geom. 78 (2008), 295-332. MR.2394025

12. X. Wang, On conformally compact Einstein manifolds, Math. Res. Lett. 8 (2001), 671-688. MR.1879811 (2003d:53075)

13. E. Witten and S. T. Yau, Connectedness of the boundary in the AdS/CFT correspondence, Adv. Theor. Math. Phys. 3 (1999), 1635-1655. MR1812133(2002b:53071)

14. S. T. Yau, Harmonic functions on complete Riemannian manifolds, Comm. Pure Appl. Math. 28 (1975), 201-228. MR0431040 (55:4042)

Division of Mathematics, National Center for Theoretical Sciences (South), Department of Mathematics, National Cheng-Kung University, Tainan 701, Taiwan

E-mail address: khlam@alumni.uci.edu 